\title{
Pursuing Cancer Research with Open Mind and Open Journal
}

\author{
Xiaoming $\mathrm{Li}^{*}$ \\ Professor and Director, Department of Otolaryngology Head Neck Surgery, Bethune International Peace Hospital, China \\ ${ }^{\star}$ Correspondence author: Xiaoming Li, Professor and Director, Department of Otolaryngology Head Neck Surgery, Bethune International Peace Hospital, China; \\ E-mail:xmlmo@126.com
}

Received: August 17, 2016; Accepted: September 17, 2016; Published: October 02, 2016

\section{Editorial}

Cancer represents one of the most threatening diseases in mankind. It is hazardous enough to endanger the health and lives of a vast population around the world, and constitutes the major cause of death in human. The clinicopathologic features of the disease vary greatly depending on the sites of origin, cell type, tumor staging and even gender, and the response to each treatment modality also differs among cancers and individuals. Establishing personalized treatment regimens for achieving the longest survival with supreme quality of life is the ultimate goal of cancer research and treatment. However, the treatment results of patients suffering from cancer are far from satisfaction because of heterogeneity in response to different therapeutic regimens by tumors. In this regard, more in-depth and long-lasting investigations are definitely needed for overcoming this embarrassing situation.

Year around the year, numerous investigators plunge themselves into the work of cancer research to unveil the most intriguing features of cancer, which provides impetus for innovating concepts and treatment strategies in the management of the disease. So far, a lot of progresses have been made in different aspects of cancer study. For instances, investigations on cancer microenvironments have disclosed some of the most mysterious parts in the pathogenesis and progression, therapeutic resistance, recurrence and metastasis of cancer; studies on molecular biology of miscellaneous cancers prompted targeted therapies by finding some specific marker proteins or molecules in the cancer cell signaling; understandings of clinicopathologic characters of different cancers greatly improved the strategic planning for surgical or non-surgical treatment; introduction of new concepts, strategies, therapeutic agents and special techniques such as induction chemotherapy, concurrent chemoradiotherapy, immnunotherapy and targeted therapies, etc. improved the treatment results and outcomes of assorted human cancers; development of new therapeutic agents added much to the multidisciplinary treatment of malignancies arising in different parts of the body. Lately, precision medicine is likely to be playing important roles in directing cancer research.

Based on our understandings and achievements that have been made in cancer prevention and treatment, it is reasonable to believe that we are getting closer to the goal of curing cancer. However, when we open arms to embrace the bright future of cancer research, we have to realize that there is still a lot to do and a long way to go before we can conquer this horrible disease. Recurrence, metastasis and resistance to various treatment modalities remain to be major challenges, which obstacle the treatment planning and strategic decisions for tumor control, and thus the treatment results and outcomes of various cancers, especially for tumors in the advanced stages. Besides, the mechanisms behind these sophisticated, uncontrollable and notorious biological behaviors are not fully understood. To solve these problems, a broad spectrum of topics and related issues have to be taken into consideration in cancer investigation, most important of which are novel concepts, new methods, new regimens, new therapeutic agents, and alternative approaches for early detection and intervention of cancer. These specific areas are all major focuses of Cancer Studies and Therapeutics (CST).

When pursuing cancer research, we do need a transparent and easy-reaching platform, such as an authentic open access scientific journal, for presenting, exchanging, searching and transmitting new ideas, concepts and methodologies associated with cancer prevention and treatment without any limitation and barrier. Meanwhile, it is necessary to adhere to the principle of open mind, open science and open journal. Our primitive motivation for launching this journal, CST, is to deliver the best communication on the fast moving, and continually evolving, global oncology landscape. To merit this peculiar motivation, we will be aiming at making this journal a real open access platform, capable of providing swift, worldwide, totally free access to the full content of every distributed article without any charge to readers or their institutions for access. Under this policy, readers are authorized to retrieve, read, download, copy, print, disseminate, or link to the full-texts of all articles published in CST.

To be frank, CST is a new member of the journal family relating to cancer research, and there is pretty much to do to develop CST into a scientific journal with high impact and reputation. Looking into the list of the editorial board members of CST, we immediately notice that they are all prominent experts with vast experience in different areas of cancer research, prevention and treatment. As the editor-inchief, I am confident that, with the rigorous, tight and harmonious cooperation among the all prestigious editorial board members, we can make CST a prosperous and fast-going scientific periodical. It is anticipated, without any doubt, that CST will be becoming a real open access platform with truly open mind and open policy, for presenting, exchanging and circulating new ideas, concepts, regimens, and treatment modalities in the scope of cancer research.

Citation:

Xiaoming Li (2016) Pursuing Cancer Research with Open Mind and Open Journal. Can Stu The J Volume 1(1): 1-1 